\title{
Sclerosing cholangitis associated with retroperitoneal fibrosis: a case of multisystem fibroinflammatory disorder
}

\author{
Grazyna T. Rompa • Anna K. Jablonska • \\ Marek T. Guzek • Michal M. Dubowik • \\ Iwona E. Marek • Marian K. Smoczynski
}

Received: 3 May 2010/Accepted: 18 September 2010/Published online: 7 October 2010

(c) The Author(s) 2010. This article is published with open access at Springerlink.com

\begin{abstract}
Introduction Retroperitoneal fibrosis is a fibroinflammatory disease of unknown etiology that can be associated with other fibroinflammatory disorders. Lately, there have been several reports showing that retroperitoneal fibrosis is a manifestation of IgG4-related sclerosing disease, which often presents as autoimmune pancreatitis.

Case report This report shows the case of IgG4-related sclerosing cholangitis associated with retroperitoneal fibrosis independent of autoimmune pancreatitis. The patient presented with cholestatic jaundice and picture of sclerosing cholangitis in endoscopic retrograde cholangiography. The combination of endoscopic biliary drainage and steroid therapy was an effective treatment in this case. Conclusion The diagnosis of $\mathrm{IgG} 4-$ related sclerosing disease should always be taken into consideration in patients with sclerosing cholangitis, especially when it is associated with any fibroinflammatory disorder.
\end{abstract}

Keywords Sclerosing cholangitis - Retroperitoneal fibrosis - Multisystem fibroinflammatory disorder . IgG4-related sclerosing disease

\section{Introduction}

Retroperitoneal fibrosis (also known as Ormond's disease) is a rare disorder characterized by the presence of fibroinflammatory tissue in the retroperitoneal space, which

G. T. Rompa ( $\square)$ · A. K. Jablonska · M. T. Guzek ·

M. M. Dubowik · I. E. Marek · M. K. Smoczynski

Department of Gastroenterology and Hepatology,

Medical University of Gdansk, ul. Debinki 7,

80-211 Gdansk, Poland

e-mail: grazina@gumed.edu.pl usually surrounds the abdominal aorta and iliac arteries [1]. The pathogenesis of the disease remains unclear. It seems that autoimmune mechanisms play a crucial part in the induction of chronic inflammation and fibrosis. It is difficult to state whether it is a localized process or just a manifestation of a systemic disease. The frequent association of retroperitoneal fibrosis with autoimmune diseases (e.g., systemic lupus erythematosus, rheumatoid arthritis, vasculitis, autoimmune thyroid diseases) and fibrosis of additional organs (e.g., sclerosing cholangitis, orbital pseudotumor, Riedel's thyroiditis) suggest the systemic mechanism of the disease. Therefore, retroperitoneal fibrosis is regarded by some authors as one of the manifestations of a multisystem fibroinflammatory disorder [2].

Lately, a new term "IgG4-related sclerosing disease" has been proposed to describe the group of autoimmune disorders involving connective tissue. Kamisawa et al. have demonstrated IgG4 antibody-stained plasma cells in a number of organs and proposed a new clinicopathological entity of IgG4-related autoimmune diseases. The authors suggested that stricture of the bile duct, sialadenitis or autoimmune pancreatitis were induced by extensive IgG4-positive plasma cell infiltration of organs, together with CD4- or CD8-positive T-lymphocytes. Clinical manifestation is apparent in the organs in which tissue fibrosis is pathologically induced and it is characterized by clinical improvement on steroid therapy [3]. The infiltration of IgG4-positive plasma cells and T-lymphocytes has been observed in various organs: pancreas, bile ducts, gallbladder, salivary glands, retroperitoneum, kidneys, lungs and prostate. Most manifestations of IgG4-related sclerosing disease are associated with autoimmune pancreatitis, but there are also cases without pancreas involvement as in the case of our patient [4-7].

There are few case reports of Ormond's disease with involvement of the bile ducts. In most of them, the 
common bile duct was encased [8-15]. Only single cases presented retroperitoneal fibrosis with involvement of the intrahepatic bile ducts and the image of sclerosing cholangitis [16-18]. Diagnosing IgG4-related sclerosing cholangitis remains a great clinical challenge, especially if is not associated with autoimmune pancreatitis. It needs to be differentiated from primary sclerosing cholangitis (PSC) and cholangiocarcinoma, as it results in completely different therapeutic strategy. Such diagnosis should always be taken into account in patients with cholangiographic picture of sclerosing cholangitis, especially if it coexists with any other potential IgG4-related disease. Diagnostic difficulties lead to unnecessary surgery in many patients and some of them may be left without an appropriate therapy. This report shows the case of IgG4-related sclerosing cholangitis associated with retroperitoneal fibrosis independent of autoimmune pancreatitis.

\section{Case report}

A 51-year-old man was admitted to the Department of Gastroenterology and Hepatology of the Medical University in Gdansk in January 2007 because of increasing jaundice of a few weeks, severe pruritus, fatigue, loss of appetite and weight loss up to $30 \mathrm{~kg}$ in the 4 preceding months.

His medical history was significant for Ormond's disease diagnosed 2 years earlier. The disease presentation at that time was acute renal failure due to obstructive uropathy. Other etiologic factors of retroperitoneal fibrosis were excluded and steroid therapy was applied with good clinical effect. Total $\operatorname{IgG}$ level was elevated at that time, $27.44 \mathrm{~g} / \mathrm{L}(5.4-20.34 \mathrm{~g} / \mathrm{L})$, but no assessment of IgG4 level was performed. The patient had also suffered from hypothyroidism due to Hashimoto's thyroiditis for 26 years and arterial hypertension for 10 years. He was using the following medications: methylprednisolone $4 \mathrm{mg}$ daily, perindopril and levothyroxine $100 \mu \mathrm{g}$ daily.

Physical examination revealed jaundice, skin excoriations and hepatomegaly. There was no tenderness of the abdomen.

In the initial laboratory tests, the following pathologies were observed (reference values in parentheses): anemiahemoglobin level $111 \mathrm{~g} / \mathrm{L}(140-180 \mathrm{~g} / \mathrm{L})$, erythrocyte sedimentation rate $58 \mathrm{~mm} / \mathrm{h}(2-20 \mathrm{~mm} / \mathrm{h})$, total bilirubin $589.95 \mu \mathrm{mol} / \mathrm{L}(3.4-20.5 \mu \mathrm{mol} / \mathrm{L})$, alkaline phosphatase $228 \mathrm{U} / \mathrm{L}(40-150 \mathrm{U} / \mathrm{L})$ and the presence of anti-HCV antibodies (later confirmed with HCV RNA test). Hepatitis B was excluded. Electrolytes, coagulation tests, C-reactive protein, and glucose and liver function tests other than alkaline phosphatase were within normal limits. The patient was also tested for autoimmune liver diseases: antineutrophil cytoplasmic antibodies (ANCA), smooth muscle cells antibodies (SMA) and antimitochondrial antibodies (AMA) were within normal limits; antinuclear antibodies (ANA) were elevated to $320(<1: 40)$ and bile duct antibodies (BDA) to $20(<1: 5)$, which were not specific. Total IgG level was normal on admission, but an elevated concentration of IgG4 (at $2.46 \mathrm{~g} / \mathrm{L}[0.03-2.0 \mathrm{~g} / \mathrm{L}]$ ) was noted during hospitalization. Apart from that, an increase in thyroidstimulating hormone (TSH) serum level to $36.38 \mu \mathrm{U} / \mathrm{mL}$ $(0.34-4.94 \mu \mathrm{U} / \mathrm{mL})$ and a decrease in free thyroxine (T4) serum level to $10.18 \mathrm{pmol} / \mathrm{L}(9.01-19.05 \mathrm{pmol} / \mathrm{L})$ was observed on admission to the hospital. The dose of levothyroxine was increased to $150 \mu \mathrm{g} /$ daily with good clinical effect (after a few months, TSH level was $5.6 \mu \mathrm{U} / \mathrm{mL}$ and T4 was $14.7 \mathrm{pmol} / \mathrm{L}$ ).

Abdominal ultrasonography showed hepatomegaly, dilated intrahepatic bile ducts and no signs of cholelithiasis, tumor of pancreas or liver hilus. Abdominal CT revealed hepatomegaly with marked cholestasis in both hepatic lobes, thickening of the gallbladder wall and a 5$\mathrm{mm}$ mantle of soft tissue along the ventral side of the aorta that reflected sclerosing changes in the course of Ormond's disease (Fig. 1a, b).

Endoscopic retrograde cholangiopancreatography (ERCP) was performed, which revealed segmental strictures of the right hepatic duct with post-stenotic dilation (Fig. 2). There were no stones in the bile duct. The main stricture of the right hepatic duct was mechanically dilated and nasobiliary drain and biliary stent were placed into the right hepatic lobe. The pancreatic duct was normal.

In the course of therapy, repeated lavage of bile ducts with saline solution was performed and corticosteroids were administered (first methylprednisolone intravenously and then prednisone orally). The patient also received ursodeoxycholic acid. In a bile culture, Streptococcus viridans was present and amoxicillin + clavulanic acid was administered.

After 9 days of hospital stay, fever over $38.5^{\circ} \mathrm{C}$ appeared and cholangitis was diagnosed. Bile culture revealed Acinetobacter baumanii infection and the antibiotic was changed to imipenem (according to microbial sensitivity test). Another ERCP was performed, which showed multifocal strictures of intrahepatic bile ducts. Nasobiliary drain in the right hepatic duct was replaced with a stent, and a drain and a stent were placed into the left hepatic duct. The bile ducts' lavage, antibiotics and corticosteroids were continued with good clinical effect.

After 7 weeks of biliary lavage and steroid therapy, total bilirubin level was $32.49 \mu \mathrm{mol} / \mathrm{L} \quad(3.4-20.5 \mu \mathrm{mol} / \mathrm{L})$, gamma-glutamyltransferase was $190 \mathrm{U} / \mathrm{L}$ (8-61 U/L), and other liver enzymes and laboratory tests were within normal limits. During ERCP, nasobiliary drain was replaced with a biliary stent. Follow-up CT revealed only moderate intrahepatic cholestasis (Fig. 3). Methylprednisolone dose was gradually tapered. The biliary stent was replaced after 
Fig. 1 CT showing

hepatomegaly with cholestasis in both hepatic lobes (a) and mantle of soft tissue along the ventral side of aorta-arrow (b)
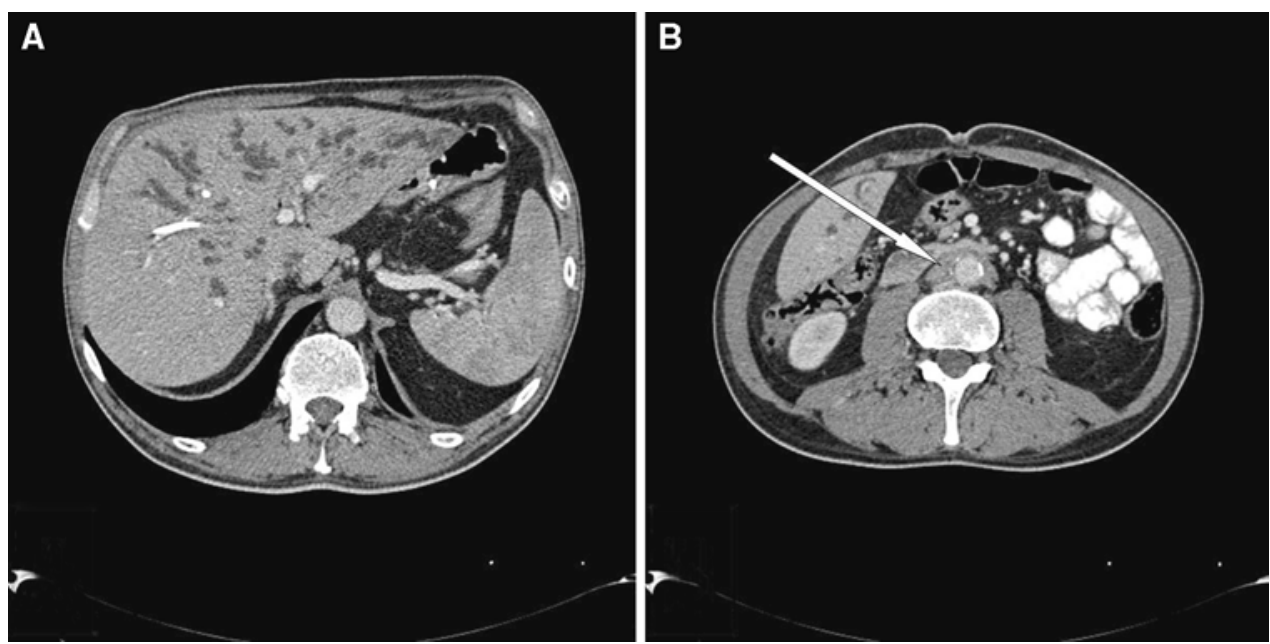

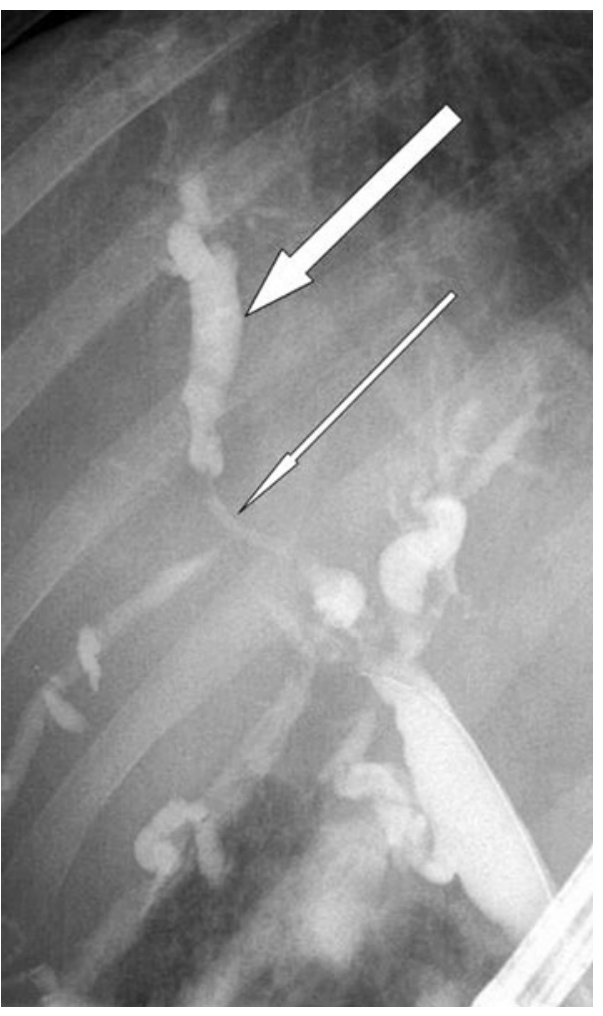

Fig. 2 ERCP showing segmental strictures (thin arrow) of the right hepatic duct with post-stenotic dilation (thick arrow)

6 months, at which time the patient's general condition was good and laboratory tests were unremarkable.

Another abdominal CT performed in April 2008 revealed a significant decrease of intrahepatic cholestasis in comparison to the CT from January 2007. There was only a moderate dilation of bile ducts in the left hepatic lobe and in the hilar region. In July 2008, ERCP was performed, biliary stents were removed and no strictures of bile ducts were visible. The patient was feeling well; physical examination and laboratory tests were unremarkable. Another abdominal

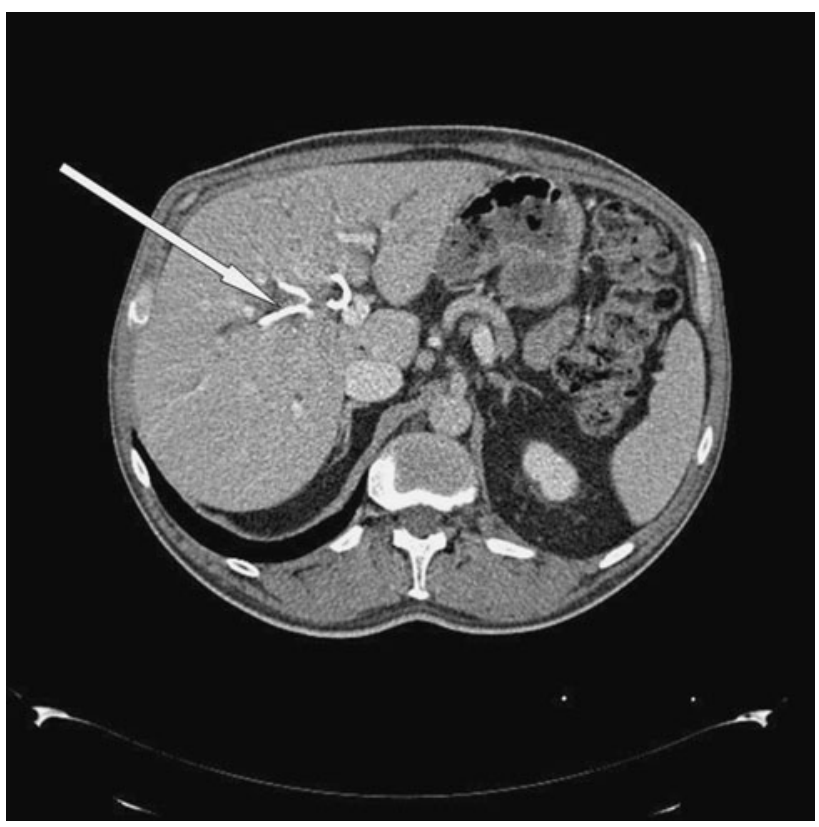

Fig. 3 CT showing moderate intrahepatic cholestasis in both lobes; stents in hepatic bile ducts (arrow)

CT in September 2009 revealed complete regression of both cholestasis and pathological mass around the abdominal aorta (Fig. 4a, b). At the 3-year follow-up, the patient is doing well with no signs of disease relapse and continues to take methylprednisolone $8 \mathrm{mg}$ daily.

\section{Discussion}

Most reports presenting retroperitoneal fibrosis associated with the involvement of bile ducts were related to cases of obstruction of the common bile duct at the level of the pancreatic head due to autoimmune pancreatitis [8-15]. In those case reports, clinical picture and imaging studies 
Fig. 4 CT showing complete regression of both cholestasis (a) and pathological mass around the abdominal aorta (b)
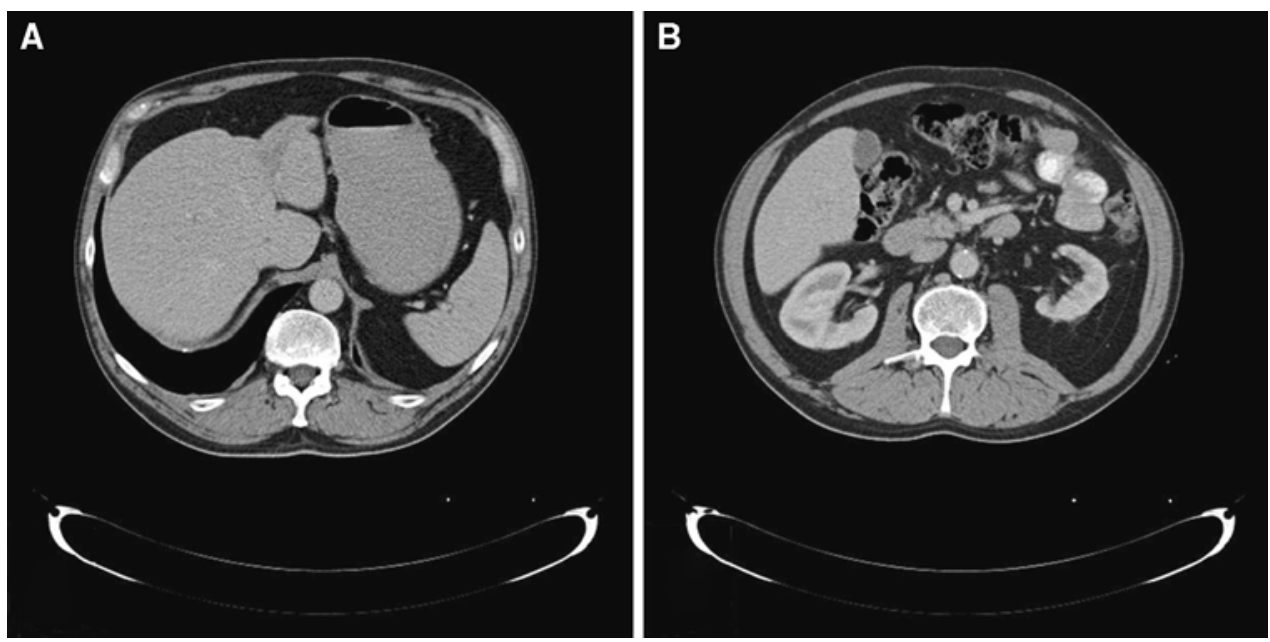

suggested the diagnosis of pancreatic cancer or cholangiocarcinoma, and the patients underwent surgical treatment with histological assessment. In the case of our patient, endoscopic retrograde cholangiography revealed multiple strictures of intra- and extrahepatic bile ducts, as in sclerosing cholangitis. Similar cases of sclerosing cholangitis associated with retroperitoneal fibrosis were previously reported by Bartholomew in the 1960s and Laitt in the 1990s [19, 20]. Recently, there have been a few reports of Ormond's disease associated with sclerosing cholangitis. In those patients, elevation of $\mathrm{IgG}$ and $\mathrm{IgG} 4$ level was observed, as well as coexistence of other autoimmune diseases such as sclerosing pancreatitis, sialadenitis and Sjögren's syndrome [16-18]. In 2007, a new term "IgG4associated cholangitis" (IAC) has been introduced referring to the biliary manifestation of IgG4-related systemic disease [6, 21]. In 2008, Ghazale presented the HISORt criteria for diagnosis of IAC [21]. In cases in which features of sclerosing cholangitis are visible on cholangiography, the diagnosis of both PSC and IAC should be considered. Both PSC and IAC are characterized by intraand extrahepatic strictures of the bile ducts; therefore, cholangiography is similar in both disorders and is not a differentiating diagnostic tool $[22,23]$. Patients with IAC respond to steroid therapy, while patients suffering from PSC are resistant to steroids and eventually need liver transplant. In contrast to PSC, IAC is not associated with inflammatory bowel diseases, but it usually coexists with fibroinflammatory changes in other organs such as pancreas, salivary glands, retroperitoneal space, lymph nodes and kidneys. IAC is most often associated with autoimmune pancreatitis, but pancreatic disease is not essential for the diagnosis of IAC [4, 21, 22]. IAC is characterized by elevation of serum IgG4 level and lymphoplasmacytic infiltration (with $>10$ IgG4-positive cells/hpf) on histological examination inside or around the bile ducts $[7,23,24]$.
In the case of our patient, ERCP revealed multiple segmental strictures of intrahepatic bile ducts that could occur both in PSC and IAC. Good response to steroid treatment, association with other fibroinflammatory diseases such as retroperitoneal fibrosis and Hashimoto thyroiditis, elevation of serum $\mathrm{IgG}$ at the moment of diagnosis of retroperitoneal fibrosis and IgG4 in the course of therapy of sclerosing cholangitis suggest the diagnosis of IAC. There was no need for surgery and no biopsy of bile ducts was performed. However, even without histological examination, taking into account all the data mentioned above, we can classify our patient into the group of high index of suspicion of IAC and we can exclude PSC. Sclerosing pancreatitis associated with retroperitoneal fibrosis is described by many authors as "autoimmune pancreatitis" due to multisystem fibroinflammatory disorder or IgG4-related sclerosing disease [5, 14]. Therefore, it seems that the cases of sclerosing cholangitis due to retroperitoneal fibrosis, as in the patient described above, are most probably the biliary manifestation of steroid-responsive multisystem fibroinflammatory disorder [5]. It is important to single out those patients and differentiate IAC from PSC, because of completely different therapeutic strategies. In the case of our patient, the combination of steroid therapy and endoscopic drainage of the bile ducts was performed to reduce the very high bilirubin level as fast as possible, and to prevent the potential fatal consequences of very high hyperbilirubinemia and cholangitis. Ghazale et al. had also performed biliary stenting to fasten the resolution of jaundice [21]. The question remains how long the treatment should be continued. There are no data on what the duration of IAC therapy should be. Maybe, the repeated assessment of serum IgG4 level could be a useful marker for monitoring and planning the continuation of treatment.

In the case of our patient, we can also discuss whether there was an association between sclerosing cholangitis and Hashimoto thyroiditis, or if they occurred 
coincidentally. Recently, Li et al. have found similar histopathological features to IgG4-related sclerosing disease in the fibrous variant of Hashimoto autoimmune thyroiditis. The authors proposed a new classification by which Hashimoto autoimmune thyroiditis can be subclassified into two groups: IgG4 thyroiditis and non-IgG4 thyroiditis. IgG4 thyroiditis may be closely related to IgG4-related sclerosing disease $[25,26]$. There was no need for biopsy of the thyroid gland in our patient; therefore, we cannot prove that it was an IgG4 thyroiditis. However, the exacerbation of hypothyroidism at the time of diagnosis of IAC and the need for an increase of levothyroxine dose after many years of stable thyroid disease might suggest some association between those two autoimmune disorders.

The diagnosis of IgG4-related sclerosing disease should always be taken into consideration in patients with sclerosing cholangitis, especially when it is associated with any fibroinflammatory disorder. In the case of the patient presented above, the combination of repeated and complicated endoscopic biliary drainage with steroids turned out to be an effective and complementary therapeutic strategy.

Open Access This article is distributed under the terms of the Creative Commons Attribution Noncommercial License which permits any noncommercial use, distribution, and reproduction in any medium, provided the original author(s) and source are credited.

\section{References}

1. Vaglio A, Salvarani C, Buzio C. Retroperitoneal fibrosis. Lancet 2006;367:241-251

2. Van Bommel EF. Retroperitoneal fibrosis. Neth J Med 2002;60:231-242

3. Kamisawa T, Funata N, Hayashi Y, Eishi Y, Koike M, Tsuruta K, Okamoto A, Egawa N, Nakajima H. A new clinicopathological entity of IgG4-related autoimmune disease. J Gastroenterol 2003;38:982-984

4. Zen Y, Harada K, Sasaki M, Sato Y, Tsuneyama K, Haratake J, Kurumaya H, Katayanagi K, Masuda S, Niwa H, Morimoto H, Miwa A, Uchiyama A, Portmann B, Nakanuma Y. IgG4-related sclerosing cholangitis with and without hepatic inflammatory pseudotumor, and sclerosing pancreatitis-associated sclerosing cholangitis: do they belong to a spectrum of sclerosing pancreatitis? Am J Surg Pathol 2004;28:1193-1203

5. Kamisawa T, Okamoto A. Autoimmune pancreatitis: proposal of IgG4-related sclerosing disease. J Gastroenterol 2006;41: 613-625

6. Björnsson E, Chari ST, Smyrk TC, Lindor K. Immunoglobulin G4 associated cholangitis: description of an emerging clinical entity based on review of the literature. Hepatology 2007; 45:1547-1554

7. Kamisawa T, Okamoto A. IgG4-related sclerosing disease. World J Gastroenterol 2008;14:3948-3955

8. Renner IG, Ponto GC, Savage WT, Boswell WD. Idiopathic retroperitoneal fibrosis producing common bile duct and pancreatic duct obstruction. Gastroenterology 1980;79:348-351

9. Remedios D, Coppen M, Bradbeer J, Theodossi A. Chronic periaortitis presenting as common bile duct obstruction. Gut 1991;32:713-714
10. Cappell MS. Obstructive jaundice due to retroperitoneal fibrosis involving the head of the pancreas. J Clin Gastroenterol 1994; 18:53-56

11. Chutaputti A, Burrell MI, Boyer JL. Pseudotumor of the pancreas associated with retroperitoneal fibrosis: a dramatic response to corticosteroid therapy. Am J Gastroenterol 1995;90:1155-1158

12. Pereira-Lima JC, Kromer MU, Adamek HE, Riemann JF. Cholestatic jaundice due to Ormond's disease (primary retroperitoneal fibrosis). Hepatogastroenterology 1996;43:992-4

13. Dejaco C, Ferenci P, Schober E, Kaserer K, Fugger R, Novacek G. Stenosis of the common bile duct due to Ormond's disease: case report and review of the literature. J Hepatol 1999;31: 156-159

14. Zhao MF, Tian Y, Guo KJ, Ma ZG, Liao HH. Common bile duct obstruction due to fibrous pseudotumor of pancreas associated with retroperitoneal fibrosis: a case report. World J Gastroenterol 2004;10:3078-3079

15. Quante M, Appenrodt B, Randerath S, Wolff M, Fischer HP, Sauerbruch T. Atypical Ormond's disease associated with bile duct stricture mimicking cholangiocarcinoma. Scand J Gastroenterol 2009;44:116-120

16. Kamisawa T, Nakajima H, Egawa N, Funata N, Tsuruta K, Okamoto A. IgG4-related sclerosing disease incorporating sclerosing pancreatitis, cholangitis, sialadenitis and retroperitoneal fibrosis with lymphadenopathy. Pancreatology 2006;6:132-137

17. Fukui T, Okazaki K, Yoshizawa H, Ohashi S, Tamaki H, Kawasaki K, Matsuura M, Asada M, Nakase H, Nakashima Y, Nishio A, Chiba T. A case of autoimmune pancreatitis associated with sclerosing cholangitis, retroperitoneal fibrosis and Sjögren's syndrome. Pancreatology 2005;5:86-91

18. Miura H, Miyachi Y. IgG4-related retroperitoneal fibrosis and sclerosing cholangitis independent of autoimmune pancreatitis: a recurrent case after a 5-year history of spontaneous remission. JOP 2009; 10:432-437

19. Bartholomew LG, Cain JC, Woolner LB, Utz DC, Ferris DO. Sclerosing cholangitis: its possible association with Riedel's struma and fibrous retroperitonitis. Report of two cases. N Engl J Med 1963;269:8-12

20. Laitt RD, Hubscher SG, Buckels JA, Darby S, Elias E. Sclerosing cholangitis associated with multifocal fibrosis: a case report. Gut 1992;33:1430-2

21. Ghazale A, Chari ST, Zhang L, Smyrk TC, Takahashi N, Levy MJ, Topazian MD, Clain JE, Pearson RK, Petersen BT, Vege SS, Lindor K, Farnell MB. Immunoglobulin G4-associated cholangitis: clinical profile and response to therapy. Gastroenterology 2008;134:706-715

22. Nakazawa T, Ohara H, Sano H, Ando T, Aoki S, Kobayashi S, Okamoto T, Nomura T, Joh T, Itoh M. Clinical differences between primary sclerosing cholangitis and sclerosing cholangitis with autoimmune pancreatitis. Pancreas 2005;30:20-25

23. Kamisawa T, Egawa N, Tsuruta K, Okamoto A, Funata N. Primary sclerosing cholangitis may be overestimated in Japan. J Gastroenterol 2005;40:318-319

24. Nakanuma Y, Zen Y. Pathology and immunopathology of immunoglobulin G4-related sclerosing cholangitis: the latest addition to the sclerosing cholangitis family. Hepatol Res 2007;37:S478-S486

25. Li Y, Nishihara E, Hirokawa M, Taniguchi E, Miyauchi A, Kakudo K. Distinct clinical, serological and sonographic characteristics of Hashimoto's thyroiditis based with and without IgG4positive plasma cells. J Clin Endocrinol Metab 2010;95: 1309-1317

26. Li Y, Bai Y, Liu Z, Ozaki T, Taniguchi E, Mori I, Nagayama K, Nakamura H, Kakudo K. Immunohistochemistry of IgG4 can help subclassify Hashimoto's autoimmune thyroiditis. Pathol Int 2009;59:636-641 